\title{
Snlyalt $I$.
}

Die Bebiate find Durd * bezcidfnet.

\section{A. Anorbnung nad bem 3nkalt.}

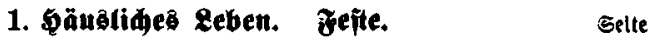

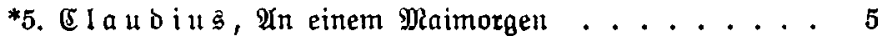

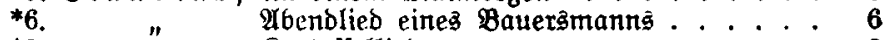

*8. " Martoffellied ............ 8

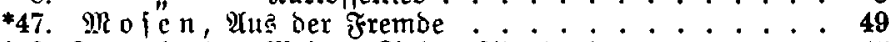

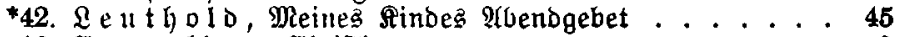

*9. E or nelius, Ehriftbnum ............ 9

*10. " Die Ĵtönige............ . . 9

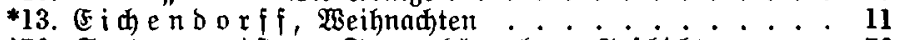

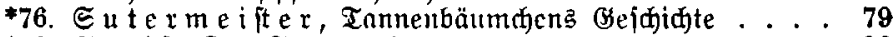

*19. B r e if, Der Sunumenomann . . . . . . . . . . . . 19

*26. Sof $f$ m a $n n$, Sonntay. . . . . . . . . . 27

*74. Ep $\mathfrak{y} i$, Morgen $i f t$ 's Sonntag ......... 77

\section{Pleisheit. Gottbertrauen.}

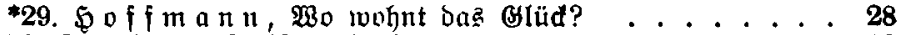

$79 \mathfrak{I}$ r o jan, Eedie unb einer .......... 83

*83. " Bum Blumenpflïden . . . . . . . . . 85

*78. " Das Pornfel ........... . . 82

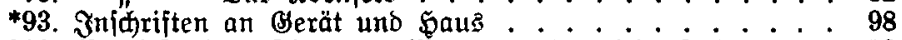

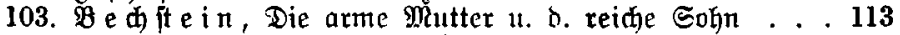

120. Br $\mathrm{r}$ m m, Die Poten des Todes ......... 155

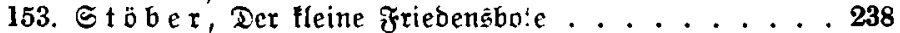

156. $\mathfrak{T} \times$ i a $\pi$, Rleinigfeiten .......... 246

185. Ein Trurt $\mathfrak{B a f f e r}$. . . . . . . . . . . . 314

186. Brocierlei $\mathfrak{A r b e i t ~ . ~ . ~ . ~ . ~ . ~ . ~ . ~ . ~ . ~ . ~ . ~ 3 1 5 ~}$

187. Bą Seneral Böben vom Betteln badte . . . . 317

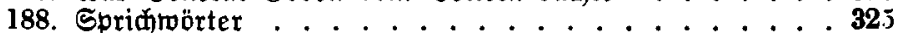

189. Beifpielpridtoörter . . . . . . . . 323

\section{Deutidie Gage und Gefidide.}

$$
\text { Sage. }
$$

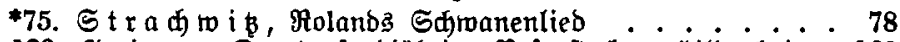

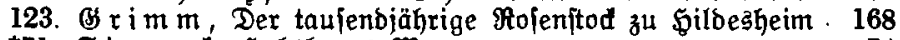

*71. S i m ro 4 , sabsburgs Mauern ......... 74

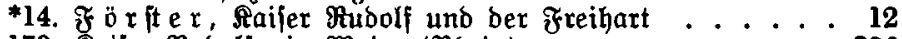

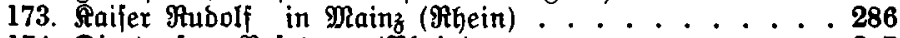

174. Die tapfere Befabung (Mhein) ......... 247

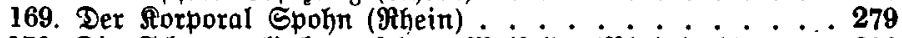

170. Die Sdrwanenfirde auf bem Maifelde (Mheinland) $\ldots 280$

171. Die Senende von ber heiligen Benoveva (Rheinland) $\ldots 281$ 
172. Drei Gagen aus bem Siebengebirge (Mhein) . . . . . 283

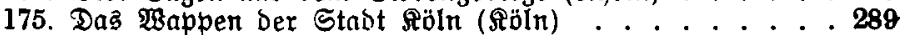

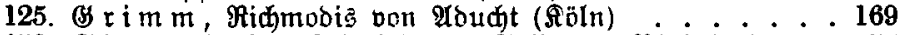

*72. S $\mathrm{i} \mathrm{m} \mathfrak{r} \mathrm{d}$, Der Sdmied bon Solingen (Mheinland) . . 75.

176. Wie bie Bupper entitand (Rlyeinland) ......290

*35. F̂ำ

*69. S4 n ezle $r$, Die Eilien im Mummelfee (Shmarzroald) 72

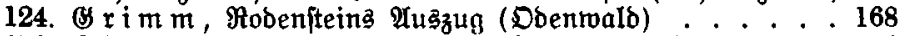

*73. S i m r od, Die 9 in ber B্Betteriahne (Frantfurt) . . 76

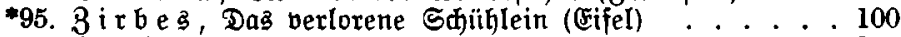

177. Der Spion von Aalen (Sdmaben) . . . . . . . . . 291

178. Der ulmer Spał (Sdruaben) . . . . . . . . . . . 293

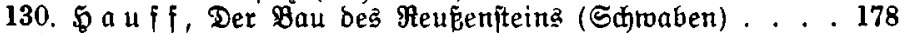

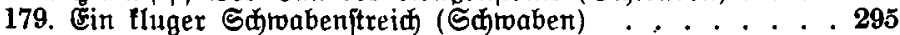

97. $\mathfrak{A} \mathfrak{u} \mathfrak{r} \mathfrak{b} \mathfrak{a}$ d e $\mathfrak{r}$, Die verroüftete $\mathfrak{A}(\mathfrak{p}$ (Dberbahern) . . . 105

180. Eulenfpiegel (:

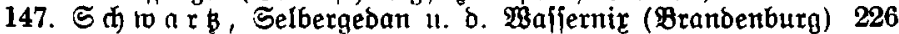

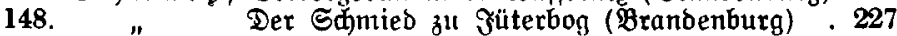

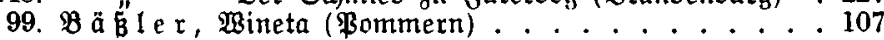

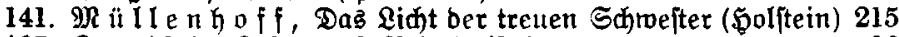

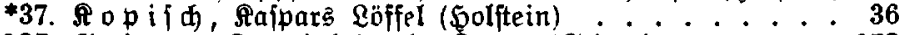

127. E $\mathrm{r}$ i m $\mathrm{m}$, Der einfelhrende $\mathfrak{Z}$ werg (Sd)

G) $e$ 占 $i$ th $t$.

*33. R e r u e r, Der reidfte F̧ürit . . . . . . . . . . 31

*43. $\&$ i $\mathfrak{g} \mathfrak{g}$, Der $\mathfrak{F}$ ahnenträger .......... 46

*20. Is re if, Die Friedengeide . . . . . . . . 19

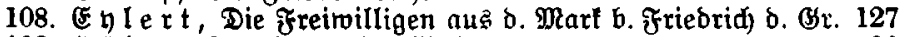

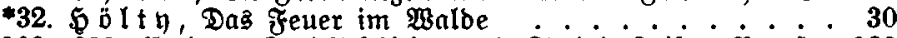

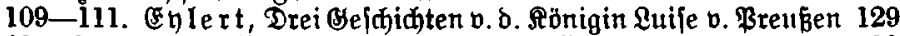

*15. ₹o o q ué, Brandenburgifuez Erntelieb ....... 13

*1. A r $n$ bt, Das Sieb vom Felomaridall ....... 1

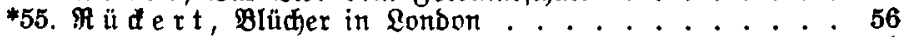

112. E $\mathfrak{l} \mathrm{l} \mathfrak{e} \mathrm{t}, \mathfrak{B o m}$ alten Geim ......... . . 133

187. Was Beneral Bäben vom Betteln bađte ..... 317

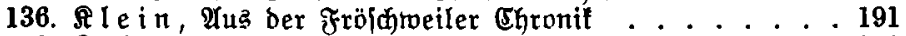

159. $3 \mathrm{e} \mathrm{i}$, Drei Rameraber ..........250

\section{Sage unb Ceidhihte atberer Bälter.}

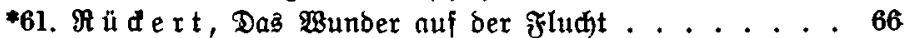

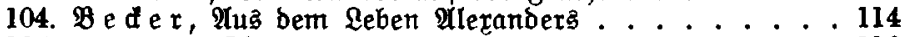
105. " Diogenes.............. 116 106. " Der Triumph des fimilius Baulus . . . 119

\section{5." Pentimes Qanb unb gort.}

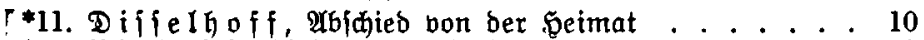

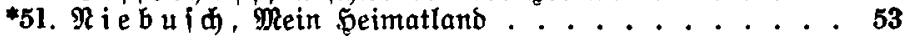

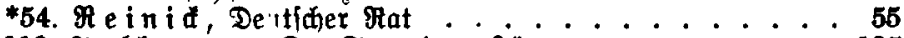

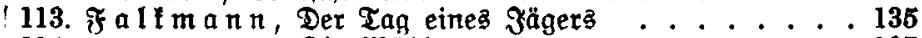

114

Die Mühle . . . . . . 137

160. Eifenbahnfahrt von Meb bis berlin $\ldots . . .253$ 
167. Die Mobelbahn ............... 271

*2. B e đa e r, Der bentide $\mathfrak{R h c i r ~ . ~ . ~ . ~ . ~ . ~ . ~ . ~ . ~ . ~ . ~} 2$

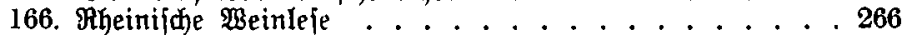

165. Trier . . . . . . . . . . . . . . 264

157. $\mathfrak{B}$ i e b i $g, \mathfrak{A m}$ Totenmaar . . . . . . . . 247

115. $\mathfrak{F} \mathrm{id}, \mathfrak{D a} \mathfrak{B}$ Buppergebiet . . . . . . . . 138

129. \&

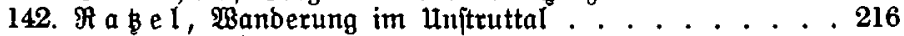

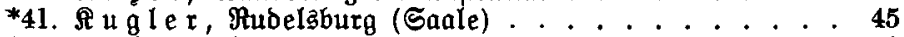

*47. $\mathfrak{M}$ o $\{$ e $\pi$, $\mathfrak{A} u$ ber frembe (Thüringen) . . . . . . 49

161. Dreşben uno bie jähfijhe Sdjweiz ........ . 257

154. Trojan, (Fijenbahnjahrt burd) bie norbbeutide Ebene. 241

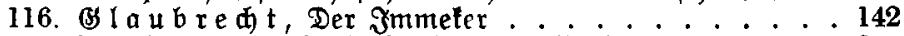

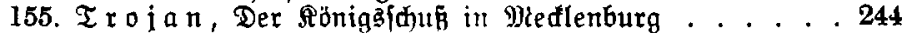

162. vamburg ............... 260

163. $\mathfrak{9}$ m Norbjeeftrande . . . . . . . . . . . . . . 262

164. Eine Galliginfel .............. . 263

96. $\mathfrak{A} \mathfrak{l} \mathfrak{m} \in \mathfrak{r} s$, Sturmflnt . . . . . . . . . 102

\section{Fuธి ber gơnembe.}

*64. S diller, fildertnabe ............ 69

*65. " arlpenjäger ............ 69

*66. $\quad$ alpenfirt . . . . . . . . . . . 69

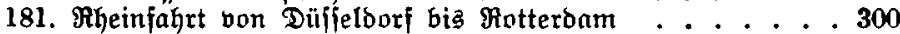

182. Daş Dorf Broet bet Amfterbam . . . . . . . 305

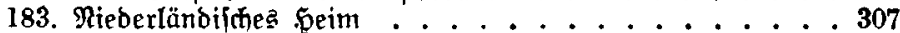

\section{Tierleben und gillangenteben.}

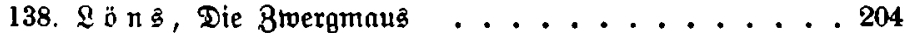

139. "Der Santor . . . . . . . 208

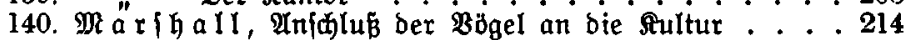

*30. S of f m a $n \mathfrak{n}$, Deg Bögleing Dant . . . . . . 28

*52. B f e ffel, Die Stufenleiter .......... 54

*91. Das Federipiel .................. 92

*90. 20adtelmadt . . . . . . . . . . . . . . . .91

144. $\Re$ u $\mathrm{B}$, Der gefangene Bauntönig . . . . . . . . 221

145. "Die Saubenlerdse ........... 223

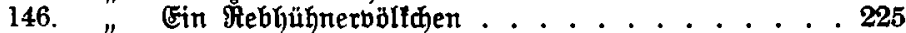

151. Se i b el, Der Bejang bes Butfifinten ......236

152. . Lerdienlieber ......... 237

*28. So fi m a $n \mathfrak{n}$, Qibellentanz $\ldots \ldots$

*59. $\Re$ ü đe $r t$, Die veripätete Piene . . . . . . . . 63

*85. T r o i a $\mathfrak{n}$, Die Räfertwage . . . . . . . . 86

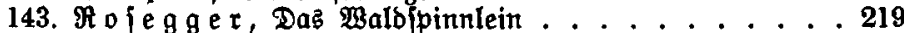

*78. T r $\mathfrak{a}$ a $\pi$, Das Somfelo .......... 82

*83. $\quad$ 8um Blumenpflüden ........ 85

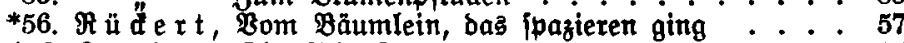

*82. I r o j a $n$, Die Sdibnfte............. 85

*44. L b to enfte in, Die wilbe Sajtanie ....... 47

*24. $\mathfrak{G}$ e $\mathfrak{b}$ e $l$, Das Sieblein bom Siríbbaum ....... 23

*40. Я r u m a d e $r$, Das sieb bom Samentorn . . . . 43 


\section{Zeben ber Exbe. Jahgreşzeiten. Zagebzeiten.}

*48. $\mathfrak{P}$ ü $1 \mathrm{l} \mathrm{e}, \mathfrak{R o h i r}$

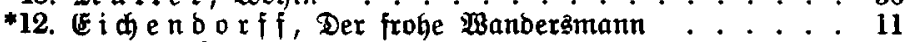

*21. \& $r$ ü $\mathfrak{n}$, 8wei Seimgefehrte .......... 20

*24. S e b e 1, Das \&ieblein nom đirjbbaum . . . . . 23

*5. ह I $\mathfrak{a} u$ b i $\mathfrak{s}, \mathfrak{A} n$ einem Maimorgen . . . . . . . . . 5

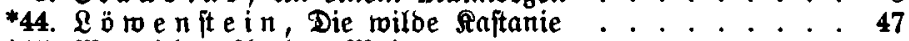

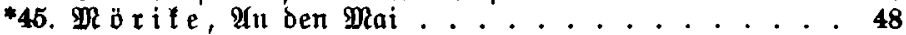

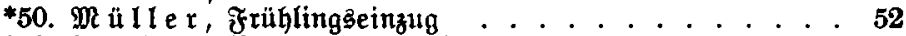

*80. Tr o j a $n$, Reues von brausen .......... 83

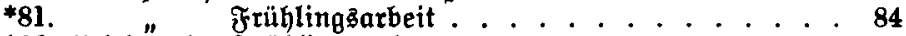

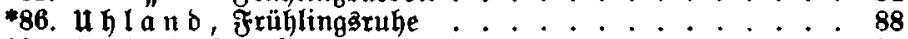

*25. \$e bel, Der Sommerabend . . . . . . . . . 25

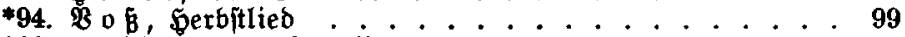

*29. So f f $m$ a $n n$, Des Bögleing Dañ ........ 28

113. I I I $\mathrm{m}$ a $n n$, Der Tag eines \}ăgers . . . . . . . 135

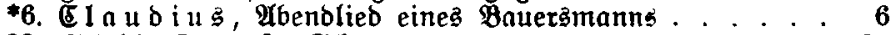

*22. \& ü II, Der erite Sqnee ............ 21

*7. ำ $а$ น

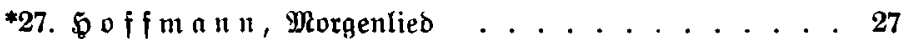

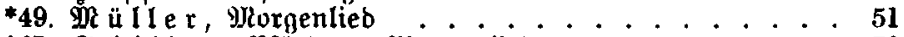

*67. S diller, Bförtners Morgenlied ........ 70

*4. M o r i $f e$, Elfenlied . . . . . . . . . . . 48

\section{B. Anoronuly nad ber รorm.}

\section{Lehrhaftes (Dibattif西es).}

\section{9. zabet. Barabel. Sprud. Mătiel.}

*52. P f e f $\mathfrak{f} \mathfrak{e l}$, Die Stufenleiter ......... 54

*58. $\Re$ ii đ e r t, Barabel: Der Ragel . . . . . . . . 63

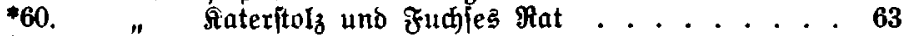

*62. " Der Perlentranz . . . . . . . . . . . . 67

189. Beifpielipriđ́wörter . . . . . . . . . . . . . . . . . . 323

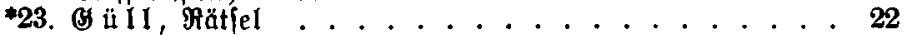

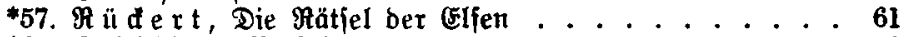

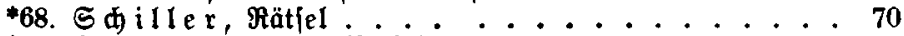

*77. Su te rme i fte $r$, Rätfel ........... 81

*92. Rätiel ............... 94

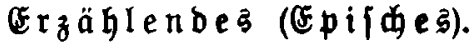

\section{Märhen.}

*56. $\Re$ ü đ̛̃ $\mathrm{e} t, \mathfrak{B}$ om Bäumlein, bas jpaz̆ieren ging . . . 57

98. $\mathfrak{A} \mathfrak{u} \mathfrak{r} \mathfrak{b}$ d $\in \mathfrak{r}$, Der Sdneiber im Mond ..... 106

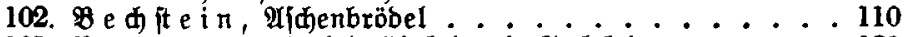

107. Bre $\tan$ no, Godel, Sintel und Badeleia ..... 121 
117. \& $\mathrm{rim} \mathrm{m}$, Sönig Droffelbart ........... 144

118. " fraul Solle ............ 148

119. " Die fluge Ellie . . . . . . . . . . 152

120. " Die Boten Des Todes ....... 155

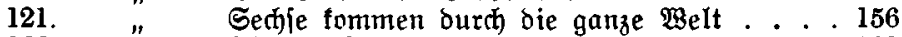

122. " Die Bänjemago ........... 162

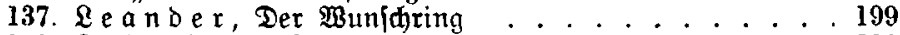

149. S e i b e l, Der Brwerg und bie Geritenähre . . . . 229

184. Dą Märden von den fieben Raben . . . . . . 310

\section{Gage. Eegende.}

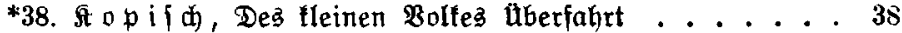

*46. $\mathfrak{M}$ ö r i $\mathrm{e}$, Elfenlied . . . . . . . . . . . . . 48

*63. Sa llet, Elfenmirtidaft .......... 67

*84. T r v i a $n$, 3werglwanderínaft .......... 85

126. $\mathrm{r} \mathrm{im} \mathrm{m}$, Die Füß̈e ber Zrverge . . . . . . . . . 170

158. W v I $\mathfrak{f}$, Der Bwerglein Silfe ........... 249

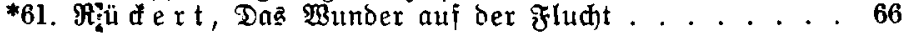

Die beutiden Sagen, bie iid an beftimmte Berfonen ober Drt= lidjteiten fnüpfen, findet man unter 3 verzeidutet.

\section{Legenden:}

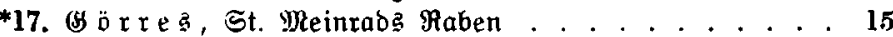

*100. Bä ä le $r$, Das $\mathscr{A}$ men ber Steine . . . . . . . . . 108

171. Die Regende von ber heiligen Benvoeva........ 281

\section{Erzählungen, ernite uno heitere, in poejie uno Broja.}

*16. BS e ib e 1 , Bon bes haifers Bart . . . . . . . . . . . 13

*18. (B) ve the Die twandelnoe Blofe . . . . . . . . . . 18

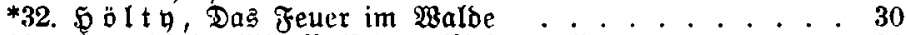

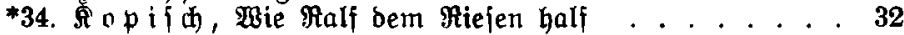

*36. " Die $28 a h r f$ it ohne Serberge . . . . . . . 35

*39. " Rleen Mämelen . . . . . . . . . 41

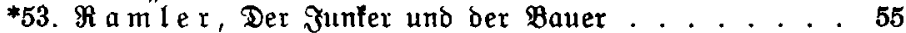

*55. $\Re$ ü đe $\mathrm{e} t$, Blüdher in Sonoon . . . . . . . . . . 56

*87. $\mathfrak{u} \mathfrak{h} \mathfrak{l} \mathfrak{a} \mathfrak{n} \mathfrak{b}$, Die $\Re a d$ e . . . . . . . . . . . . 88

101. $\mathfrak{B} \mathfrak{a} \mathfrak{B} \mathfrak{l}$ e $r, \mathfrak{B i e}$ Eullenfpiegel ein Sdneider roird . . . 109

103. B e w t e in, Die arme Mutter und ber reide ভohn . . 113

128. (s) u b e , Der \&öbe ift los! . . . . . . . . 173

131 , S e b e l, Der Star von Segringen . . . . . 181

132. " Der geheilte ßatient . . . . . . . . 183

133. " Die \$adtel ......... 185

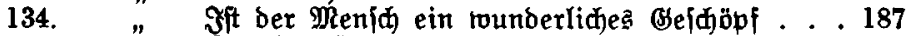

$135 . \quad$ Rannitberftan ........... 189

153. S $t$ "̆ b e $r$, Der Ileine Friebenabote . . . . 238

163. $\mathfrak{A m}$ Rorbfeeftrande . . . . . . . . . . . . 262

180. Bom Euleníbiegel .................. 297

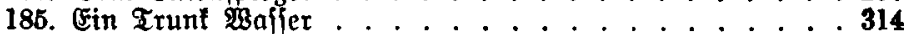

188. Spridtwörter . . . . . . . . . . . 320

189. Beifpielfpridłrörter . . . . . . . . . . . 323 
(B) efühle und Bebanten (agrijuez̃).

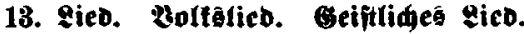

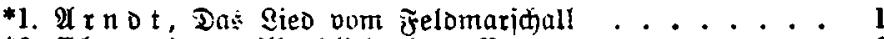

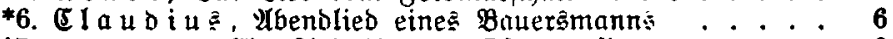

*7. " Ein Sied hinterm sien zu jingen .... 6

*8. " ftartoffellied ......... 8

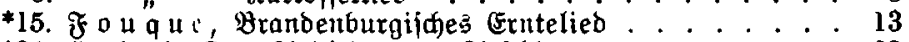

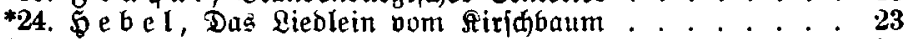

*27. So ff $m a n n$, Murgenlied ......... 27

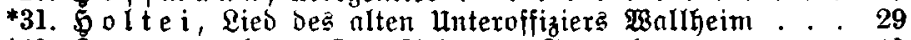

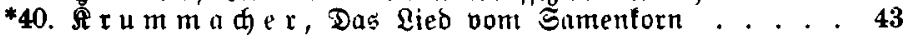

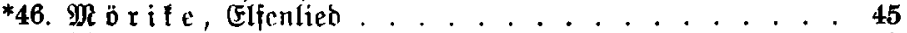

*48. $\mathfrak{R}$ ü 11 c r, $\mathfrak{B g h l i n}$ ? . . . . . . . . . 50

*49. Murgenlied .......... 48

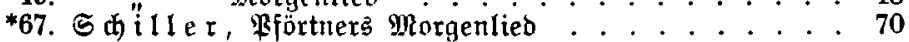

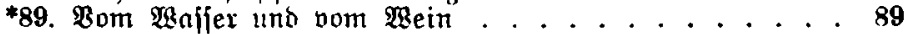

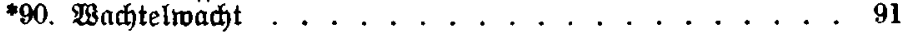

*94. $\mathfrak{B} \circ \mathfrak{B}$, serbitlied $\ldots \ldots \ldots \ldots \ldots$

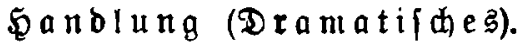

\section{Seipräđ. Gelbfigeipräd.}

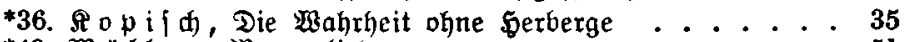

*49. M ü \ e $r$, Mrorgenlied . . . . . . . . . 51

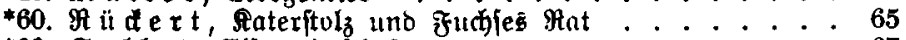

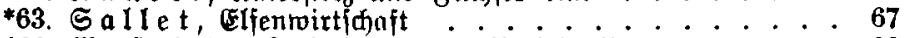

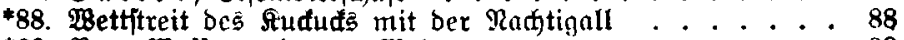

*89. Bom $28 a f f e r$ und vom

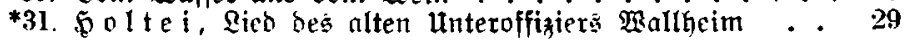

